\title{
Topical Sensor for the Assessment of PET Dose Administration: Metric Performance with an Autoinjector
}

\author{
Stephanie Sanchez ${ }^{1}$ and Geoffrey M. Currie ${ }^{2}$ \\ ${ }^{1}$ Faculty of Science, Charles Sturt University, Port Macquarie, Australia; and ${ }^{2}$ Faculty of Science, Charles Sturt University, Wagga \\ Wagga, Australia
}

\begin{abstract}
Extravasation or partial extravasation of the radiopharmaceutical dose in PET can undermine SUV and image quality. A topical sensor has been validated using several metrics to characterize injection quality after manual injection. The performance of these metrics for autoinjector administration has been assessed. Methods: A single PET/CT scanner at a single site was used to characterize injections using an autoinjector with standardized apparatus, flush volume, and infusion rate (1-min infusion followed by 2 syringe flushes) for ${ }^{18} \mathrm{~F}-\mathrm{FDG},{ }^{68} \mathrm{Ga}$-prostatespecific membrane antigen, and ${ }^{68} \mathrm{Ga}$-DOTATATE. In total, 296 patients with topical application of sensors were retrospectively analyzed using conventional statistical analysis and an artificial neural network. Results: Partial extravasation was noted in $1.3 \%$ of studies, with $9.1 \%$ (inclusive of partial extravasation) identified to have an injection anomaly (e.g., venous retention). Extravasation was independently predicted by the time that elapsed as the counts recorded by the injection sensor fell from the maximum value to within $200 \%$ of the reference sensor counts greater than $1,200 \mathrm{~s}$; as the difference in counts for injection and reference sensors, normalized by dose, from 4 min after injection greater than 25; and as the ratio of the average counts per second recorded by the injection sensor at the end of a monitoring period to those of the reference sensor greater than 2. Conclusion: Extravasation and partial extravasation of PET doses are readily detected and differentiated using time-activity curve metrics. The metrics can provide the insight that could inform image quality or SUV accuracy issues. Further validation of key metrics is recommended in a larger and more diverse cohort.
\end{abstract}

Key Words: PET; ${ }^{18}$ F-FDG; ${ }^{68}$ Ga-PSMA; extravasation; Lara

J Nucl Med Technol 2020; 48:363-371

DOI: 10.2967/jnmt.120.245043

$\mathbf{P}$

ET and the SUV, which have been well documented to change management in $38 \%$ of oncology patients (1), rely on pharmacokinetic assumptions that assume an extravasation-free injection. Extravasation occurs when part or all of the administered dose is injected outside the venous system or

\footnotetext{
Received Mar. 11, 2020; revision accepted Apr. 16, 2020.

For correspondence or reprints contact: Geoffrey M. Currie, School of Dentistry and Health Sciences, Locked Bag 588, Charles Sturt University, Wagga Wagga 2678, Australia.

E-mail: gcurrie@csu.edu.au

Published online Jun. 9, 2020

COPYRIGHT @ 2020 by the Society of Nuclear Medicine and Molecular Imaging.
}

leaks into surrounding tissue (2-4) because of errors in placement of the needle or dislodgement. Dislodgement might occur in response to injection volume, injection pressure, movement, or several patient factors. Partial extravasation of the intravenous dose administration undermines the predictability of dose delivery, image quality, and the accuracy of the SUV (2).

An examination of partial-extravasation rates in 400 patients (5) found a rate of $10.5 \%$, with $31 \%$ of those being undetected using standard imaging protocols (arms outside the field of view). A second study provided an analysis of 1,367 patient studies and found an $18 \%$ extravasation rate involving between $1 \%$ and $22 \%$ of the injected dose (6). A smaller single-center analysis found $38 \%$ of patients to have extravasated doses (7). Identifying and characterizing PET dose partial extravasation present several challenges in the clinical setting. First, the injection site is generally outside the field of view (arms up) and undetected. Second, dose administration techniques (infusion, autoinjectors) may prevent visual identification of signs of extravasation (pain and swelling) at the time of injection. Third, when partial extravasation is noted, quantifying the extent of extravasation and correcting the SUV are not readily achievable. Consequently, the studies outlined above are likely to underestimate extravasation rates and impact. A broader literature review suggests that the detectable extravasation rate (likely to be an underestimate) ranges from $9 \%$ to $23 \%$ for manual administrations (4).

A simple method for detecting and potentially characterizing PET dose extravasation is the use of topically applied scintillation sensors to monitor activity migration from the injection site during the uptake phase. The only commercially available device designed for this purpose is the Lara (Lucerno Dynamics, LLC) device. The performance of the Lara device, to detect partial-dose extravasation and provide dynamic data that assist in characterizing the extravasation, has been widely reported for manual administration of ${ }^{18} \mathrm{~F}-\mathrm{FDG}(2,4,7) .{ }^{18} \mathrm{~F}-$ FDG injections in the hand, wrist, or forearm have higher extravasation rates than injections in the antecubital fossa; higher doses increase extravasation rates, lower body weights are associated with increased extravasation, and lower flush volumes are associated with increased extravasation (4).

Generally, the administration of PET doses has not been monitored in the clinical scenario, perhaps becausecompared with CT and MRI contrast administration-the 
relative volume of the PET dose is very small, the local consequences of extravasation are benign, and direct (manual) injection is relied on. It is important in principle, and in using detection systems, to differentiate dose extravasation, extravasation with complete resolution during the uptake period, and venous retention with rapid and complete resolution. The Lara device is coupled to Lucerno Dynamics proprietary software, whose algorithm generates several metrics that guide decision making regarding dose-injection quality and differentiation between extravasation and venous retention or other anomalies.

The aim of this investigation was to evaluate the Lara quality assurance tool and associated metrics-specifically, the role of metrics for characterizing extravasation using the autoinjector method. Autoinjectors reduce contamination risk and occupational exposure of the staff to radiation; however, the injection is not supervised to detect extravasation, and the bolus profile may vary with the infusion process.

\section{MATERIALS AND METHODS}

The project was approved by the institutional ethics committee for retrospective analysis of the data from a quality improvement initiative forming standard care in a single PET/CT scanner department at a single center. Patients referred for ${ }^{18} \mathrm{~F}-\mathrm{FDG},{ }^{68} \mathrm{Ga}-$ DOTATATE, and ${ }^{68} \mathrm{Ga}$-prostate-specific membrane antigen (PSMA) PET/CT studies underwent Lara monitoring as part of their standard care. Patients were cannulated in any vein, generally the antecubital fossa. A 20-gauge needle was used unless circumstances demanded a different gauge. In each of 3 injection uptake rooms, Lara sensors were set up and applied to the subject using adhesive pads $7 \mathrm{~cm}$ proximal to the injection site and the same site on the contralateral arm (reference arm) using a previously described method (7). A KARL100 autoinjector (Tema Sinergie) was connected through the wall to the cannula, with the injection delivered to the patient via the Rad-Inject pump (Tema Sinergie) over $1 \mathrm{~min}$. The Rad-Inject pump then performed a double syringe flush at the same rate as the primary infusion (that is, the single-dose syringe was flushed twice). The total planned volume of the flush was standardized to $80 \mathrm{~mL}$. After dose administration, sensors collected data at 1-s intervals throughout the uptake phase (generally $50 \mathrm{~min}$ before the patient was escorted to the scanning room). Once removed from the patient, the recording device was connected to a computer and data uploaded to the Lucerno Dynamics platform, where it was interpreted by the software.

\section{Analysis}

The Lara data were extracted and presented as a time-activity curve, with the typical display showing the injection side in black and the reference side in red (Fig. 1). Although the Lara metrics have been previously validated for manual injections of ${ }^{18} \mathrm{~F}-\mathrm{FDG}$ using doses on the order of 370-740 MBq (10-20 mCi), several limitations associated with validity for this patient cohort demanded manual interpretation. These included the lower doses used for patient administration (185-370 MBq [5-10 mCi]), the shape and width of the curve generated by the KARL100 autoinjector administration, and the use of ${ }^{68} \mathrm{Ga}$ radiopharmaceuticals.

The manual interpretation of time-activity curves was based on understanding ideal injection time-activity curves: reference counts remaining low while the injection counts rapidly peak before rapidly declining to meet the reference levels (Fig. 1). Examination of the slope of the bolus injection on the time-activity curve as it approaches the reference sensor time-activity curve needs consideration. The time-activity curve count for the injection sensor relative to the reference sensor was also evaluated at various points during the uptake period. The time for the injection sensor to reduce to double the reference sensor count was calculated, and the areaunder-the-curve (AUC) ratios between injection and reference sensor on the time-activity curves was determined at multiple time points.

Specifically, the following metrics were calculated and analyzed (Fig. 2): aUCR10, which is the AUC ratio between the injection and reference curves at 1-10 min after injection; aUCR1, which is the AUC ratio between the injection and reference curves at 60-90 s after injection; $\mathrm{c} 1$, which is the average count per second recorded by the injection sensor at 60-90 s after injection; CEndINJ, which is the average count per second recorded by the injection sensor at the end of a monitoring period; CEndREF, which is the average count per second recorded by the reference sensor at the end of a monitoring period; tHalf, which is the average time (in seconds) required for the count recorded by the injection sensor to fall to half the previous value; tc50, which is the time (in seconds) that elapses as the count recorded by the injection sensor falls from the maximum value to within $200 \%$ of the reference sensor count (i.e., reference is $50 \%$ of value of injection curve); ndAvg1, which is the difference in counts for injection and reference sensors, normalized by dose, at 60-90 s after injection; ndAvgN, which is the difference in counts for injection and reference sensors, normalized by dose, from $4 \mathrm{~min}$ after injection; and time-activity curve score, which is a linear, weighted combination of these metrics, with the weights being determined from a logistic regression. Good injections are generally associated with a negative score, whereas scores over 200 typically indicate that part of the dose remains at the injection site.

These metrics are calculated automatically by the Lucerno Dynamics algorithm without operator input.

The statistical significance was calculated using $\chi^{2}$ analysis for nominal data and Student $t$ testing for continuous data. $F$ test ANOVA was used to determine statistically significant differences within grouped data. A $P$ value of less than 0.05 was considered significant.

\section{RESULTS}

There were 296 valid cases, with $1.3 \%$ (4) demonstrating evidence of extravasation on the time-activity curves (Fig. 3) whereas $9.1 \%$ (27), inclusive of extravasation cases, demonstrated some abnormality associated with dose administration (largely slow venous clearance in the timeactivity curve). Other key demographic data and differences among the different radiopharmaceuticals are summarized in Table 1.

No statistically significant differences were noted between normal, abnormal (venous retention), and extravasated subgroups with respect to dose $(P=0.060)$, despite normal having a mean lower dose; with respect to radiopharmaceutical $(P=0.315)$, despite a higher proportion of abnormal and extravasated studies being ${ }^{18} \mathrm{~F}-\mathrm{FDG}$; with respect to the person performing the injection $(P=0.414)$, patient weight $(P=0.529)$, age $(P=0.365)$, and sex 


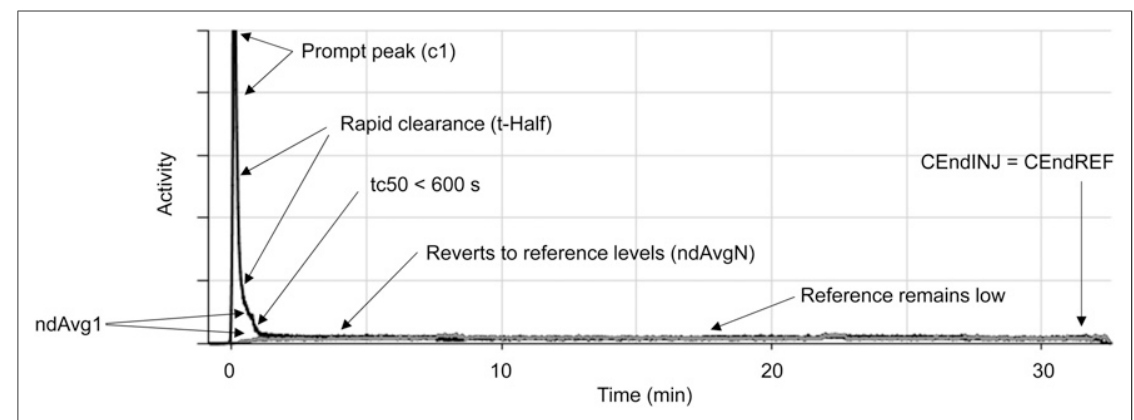

FIGURE 1. Annotated normal time-activity curve (with injection curves in black and reference curves in gray). High-count data are truncated by software to ensure that relationship between injection and reference curves is graphically discernible. Key features of normal time-activity curve include prompt peak after injection (c1), rapid clearance (tHalf), rapid reversion to reference levels (ndAvgN), and low reference level (CEndINJ compared with CEndREF). tc50, or point where injection curve is less than twice the value of the reference curve, is also less than $600 \mathrm{~s}$ (10 min), and difference between injection and reference curves at $4 \mathrm{~min}$ (ndAvg1) is low, with rapid clearance. Color version of this figure, showing gray curves as red, is available as supplemental file at http://tech.snmjournals.org.

(0.245), despite men showing a higher proportion of vascular retention and women a higher proportion of extravasation; with respect to the location of the injection $(P=0.561)$, despite there being a 2.1 times higher chance of extravasation for hand, wrist, or forearm injections over antecubital fossa; and with respect to orientation $(P=0.09)$, despite there being a higher proportion of abnormal and extravasated doses on the right side. Several statistically significant differences were noted among the calculated metrics for the different injection outcomes (Table 2). One study in which the dose leaked from the injection apparatus was classified for these purposes as extravasation, as the resulting timeactivity curve paralleled an extravasation curve. Several time-activity curves, particularly for ${ }^{68} \mathrm{Ga}$-PSMA, were truncated early, with production of an end-of-curve count anomaly that could create an aberrant CEnd ratio (Fig. 4) requiring correction of the CEndINJ and CEndREF.

Although the mean values showed discriminatory power between normal, abnormal, and extravasated doses, at an individual dose level (indicated by the range and interquartile range), few metrics provided a defined cutoff between classifications. Specifically, there was overlap of not just the range for the injection score across the injection outcomes (Table 2) but also of the interquartile range. Conversely, tc50, ndAvgN, and the newly created CEnd ratio demonstrated distinct cutoffs between extravasated injections and other injections (Table 2).
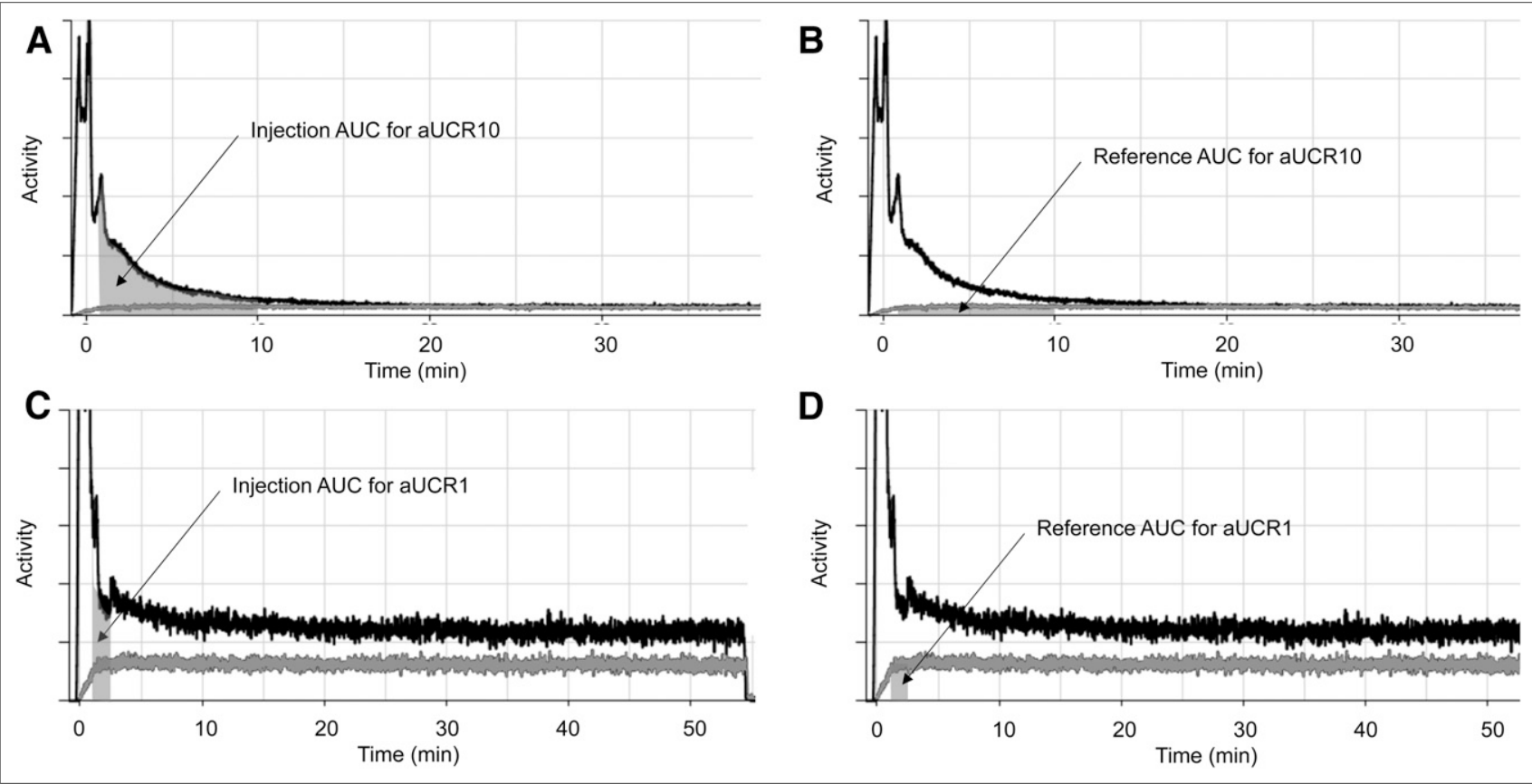

FIGURE 2. Annotated abnormal time-activity curve (with injection curves in black and reference curves in gray) indicating dose extravasation. Panel A highlights AUC for injection sensor (gray region) for aUCR10 on patient with venous retention, whereas panel $B$ highlights AUC (gray region) for reference sensor. aUCR10 is calculated as ratio of AUC from A to AUC from B. Panel C highlights $A U C$ for injection sensor (gray region) for aUCR1 in patient with extravasation of dose, whereas panel D highlights AUC (gray region) for reference sensor. aUCR1 is calculated as ratio of AUC from C to AUC from D. Color version of this figure, showing gray curves as red, is available as supplemental file at http://tech.snmjournals.org. AUC = area under the curve. 

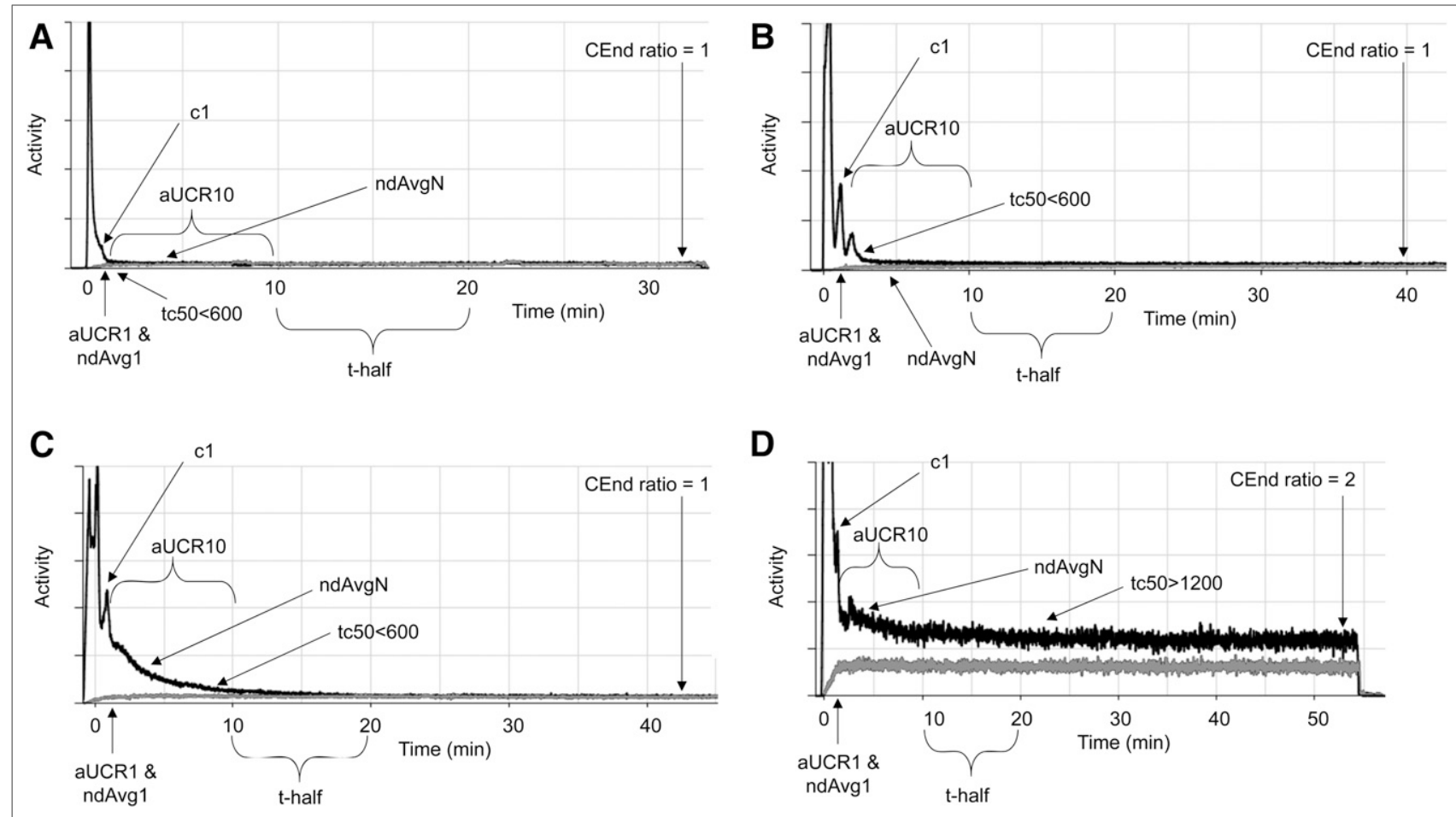

FIGURE 3. Annotated time-activity curves (with injection curve in black and reference curve in gray) for normal dose administration (A), normal administration with autoinjector multiple peaks and wider bolus (B), venous retention with resolution (C), and extravasation without resolution (D). Each time-activity curve is annotated with Lucerno Dynamics metrics automatically generated for monitored injections. Color version of this figure, showing gray curves as red, is available as supplemental file at http://tech.snmjournals.org.

The tc50 and CEnd ratio might also be used as a cutoff to indicate whether injection characteristics should be considered to influence SUV calculation. Furthermore, at the interquartile range level, ndAvgN also provided a cutoff to differentiate a normal injection from an abnormal (nonextravasated) injection with venous retention.

The previous statistical evaluation reported limitations associated with Lara metrics for autoinjector time-activity curves, including the time-activity curve score. Extravasation was independently predicted by a tc50 greater than 1,200 , ndAvgN greater than 25, and CEnd ratio greater than 2. In this evaluation, the original data were reevaluated using an artificial neural network (Neural Analyser, version 2.9.5). The purpose was to demonstrate the usefulness of machine learning as an analysis approach parallel to conventional statistical analysis $(8-10)$ and to confirm or refute observations using conventional statistical analysis.

There were 36 input variables in 296 patients, including extracted metrics, demographic data, injection parameters, and outcomes. A single binary output was an extravasated injection evidenced by the manual examination and characterization of the time-activity curve as previously described, or no extravasation (normal and other anomalies such as venous retention). A correlation matrix (heat map) was calculated to identify redundancy among variables and exclusion when appropriate. Logistic correlations revealed dependency of the outcome (extravasation) on several variables, with those of significance including tc50 (1.0), CEnd ratio (0.974), and ndAvgN (0.786), supporting the previous statistical analysis. The initial network architecture after omission of redundancy included 12 scaling layer inputs, 1 hidden layer of 3 nodes using a logistic activation function (defines the output of each node based on its input), and a single probabilistic layer (binary). The weighted squared error method was used to determine the loss index because there was an imbalance between positive and negative outputs (grounded truth). The neural parameters' normal weight was used as the regularization method to control neural network complexity. A quasi-Newtonian training method was applied, using gradient information to estimate the inverse Hessian for each iteration of the algorithm (no second derivatives). The loss function associated with the training phase estimated the error associated with the data observed by the neural network and decreased from 1.405 to 0.010 after 21 iterations. The selection loss is a measure of the neural network's agility (generalizability to new data), and in this case the error decreased from 1.452 to 0.0002 after 21 iterations. This finding indicates the need to optimize the final architecture.

A growing-input method was used to calculate the correlation for every input against each output in the dataset. Beginning with the most highly correlated inputs, progressively decreasing correlated inputs were added to the network 


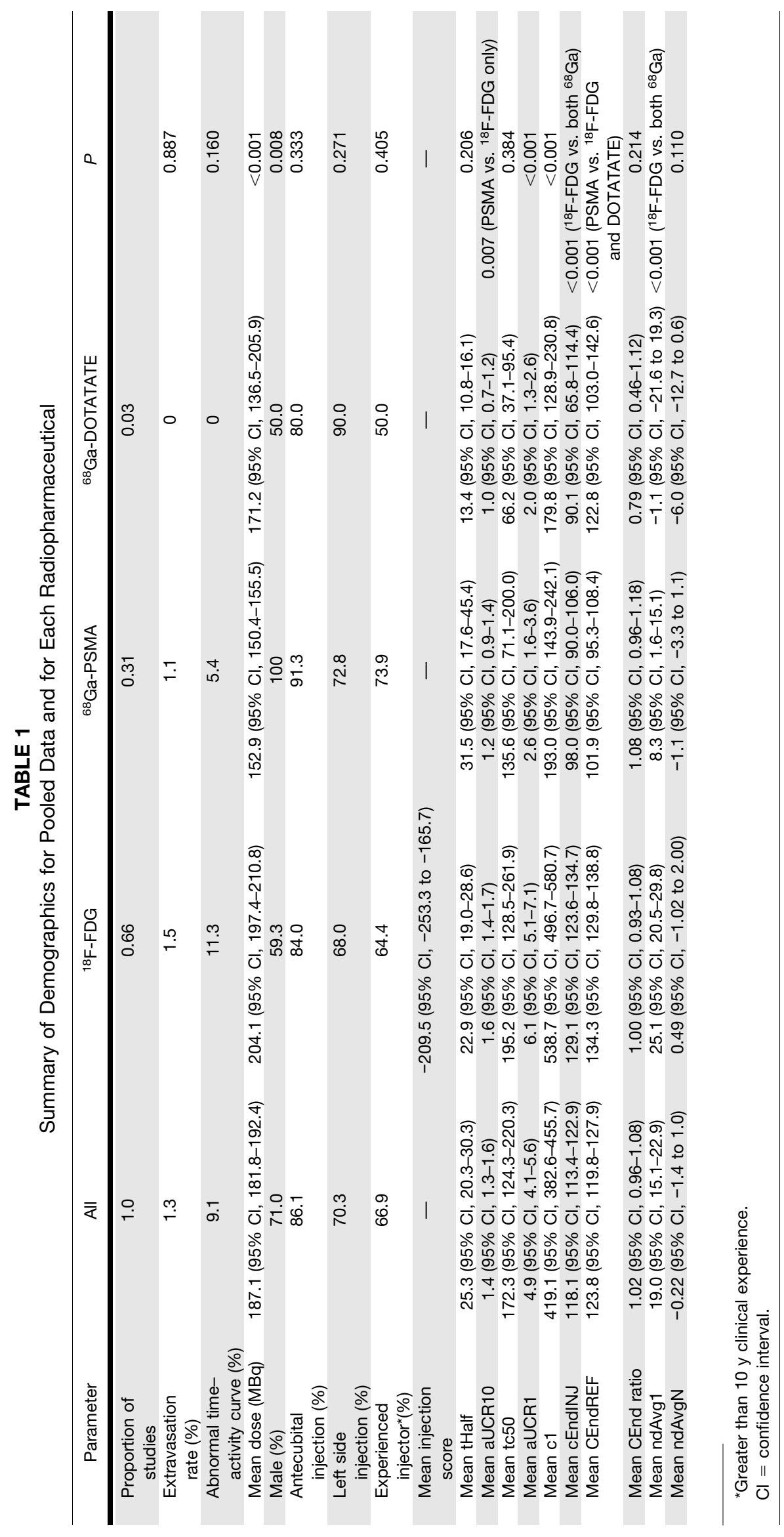


TABLE 2

Summary of Metrics Against Outcome of Injection

\begin{tabular}{|c|c|c|c|c|}
\hline Parameter & Normal & Abnormal & Extravasated & $P$ \\
\hline Description & Consistent with Figs. 3A or $3 \mathrm{~B}$ & $\begin{array}{c}\text { Consistent with Fig. 3C } \\
\text { (vascular retention) }\end{array}$ & $\begin{array}{l}\text { Consistent with Fig. 3D } \\
\text { (infiltrated) }\end{array}$ & \\
\hline Mean dose (MBq) & 185.1 & 208.6 & 199.7 & 0.060 \\
\hline Male (\%) & 70.6 & 86.4 & 60.0 & 0.245 \\
\hline Antecubital injection (\%) & 78.0 & 90.9 & 80.0 & 0.561 \\
\hline Left side injection (\%) & 72.1 & 54.6 & 40.0 & 0.090 \\
\hline Experienced injector (\%) & 68.4 & 54.5 & 40.0 & 0.414 \\
\hline Score ( ${ }^{18} \mathrm{~F}-\mathrm{FDG}$ only) & & & & $<0.001$ \\
\hline Mean & $-267(-306$ to -229$)$ & 182 (64-299) & 589 (294-885) & \\
\hline Range & $-1,056$ to $447(-343$ to -182$)$ & -256 to $1,346(-170$ to 293$)$ & -664 to 2,317 (same) & \\
\hline thalf & & & & 0.023 \\
\hline Mean & $23.1(17.9-28.3)$ & $48.4(30.2-66.6)$ & $41.3(3.1-79.4)$ & \\
\hline Range & $2.2-300(10.1-21.2)$ & $6.5-191.0(22.2-65.8)$ & $2.5-141.6(5.9-90.4)$ & \\
\hline aUCR10 & & & & $<0.001$ \\
\hline Mean & $1.2(1.1-1.3)$ & $2.7(2.4-3.1)$ & $6.7(6.0-7.3)$ & \\
\hline Range & $0.4-3.8(0.9-1.5)$ & $1.2-5.6(1.9-3.3)$ & $2.4-11.7$ (same) & \\
\hline Tc50 & & & & $<0.001$ \\
\hline Mean & $89(72-105)$ & $416(358-475)$ & $2,342(2,219-2,465)$ & \\
\hline Range & 9-956 (41-114) & $39-1,010$ (170-634) & $1,500-2,617(1,734-2,327)^{\star}$ & \\
\hline aUCR1 & & & & $<0.001$ \\
\hline Mean & $4.0(3.3-4.7)$ & $12.0(9.5-14.4)$ & $19.0(13.8-24.1)$ & \\
\hline Range & $0.6-31.5(1.7-5.0)$ & $2.5-70.1(4.5-12.7)$ & $2.9-42.3(4.1-37.3)$ & \\
\hline $\mathrm{c} 1$ & & & & $<0.001$ \\
\hline Mean & 378 (342.9-412.7) & $833(711-955)$ & $897(642-1,153)$ & \\
\hline Range & $52-1,315(163-532)$ & $211-1,577(497-1,143)$ & $198-2,110(355-1,594)$ & \\
\hline cEndINJ & & & & $<0.001$ \\
\hline Mean & $114.7(110.1-119.3)$ & $130.5(114.6-146.4)$ & $251.0(217.7-284.4)$ & \\
\hline Range & $51.8-270.2(95.1-135.7)$ & $52.5-178.0(114.2-160.4)$ & $149.9-533.4(150-385)$ & \\
\hline CEndREF & & & & 0.023 \\
\hline Mean & $125.3(121.2-129.4)$ & $121.2(107.0-135.5)$ & $83.2(53.2-113.2)$ & \\
\hline Range & 18.4-220.4 (103.8-149.1) & $36.2-163.5$ (110.9-140.7) & 69.4-123.7 (70.6-100.0) & \\
\hline CEnd ratio & & & & $<0.001$ \\
\hline Mean & $0.93(0.89-0.97)$ & $1.09(0.95-1.24)$ & $3.14(2.82-3.45)$ & \\
\hline Range & $0.34-1.82(0.78-1.07)$ & $0.7-1.5(0.9-1.3)$ & $1.9-7.0(2.0-4.8)^{\star}$ & \\
\hline ndAvg1 & & & & $<0.001$ \\
\hline Mean & $13.5(10.1-17.0)$ & $66.8(54.8-78.9)$ & $107.3(81.9-132.5)$ & \\
\hline Range & -24.9 to 108 ( -0.2 to 21.9$)$ & 8.6-203.9 (22.9-105.5) & 24.6-322.8 (38.1-202.9) & \\
\hline ndAvgN & & & & $<0.001$ \\
\hline Mean & $-1.9(-2.9$ to -0.9$)$ & $9.0(5.6-12.5)$ & $47.1(39.8-54.3)$ & \\
\hline Range & -24.8 to $11.1(-5.7$ to 2.1$)$ & -0.5 to $54.9(2.6-12.7)$ & $26.0-96.9(27.8-74.8)^{\star}$ & \\
\hline
\end{tabular}

*Distinct cutoffs between extravasated injections and other injections.

Data in parentheses are $95 \%$ confidence interval (for mean) and interquartile range (for range).

until the selection loss increased, allowing identification of the optimal number of inputs. Similarly, an incremental-order method was used for input order selection, starting with the minimum order and adding perceptrons (and complexity) until the loss increased. The final architecture of the neural network reflects the optimized subset of inputs and order with the lowest selection loss. In this case, the selection loss rose significantly for the second input, with the training loss near optimal for 1 input. Thus, the optimal number of inputs was determined to be 1 , with a training loss less than 0.001 and selection loss of 0.011 . The optimal order was also 1 (single perceptron), with a training loss of 0.013 and selection loss of less than 0.001 . The final architecture had 1 scaling-input layer (tc50), 1 hidden layer of a single node (perceptron), and a binary probabilistic output.
The final architecture was evaluated using several tests, which indicated robust validation using a subset of the original patient data. Receiver-operator-characteristic analysis demonstrated an AUC of 1.0. This correlates with a sensitivity of $100 \%$ and specificity of $100 \%$ and is reflected in the confusion matrix (100\% true-positives, $100 \%$ true-negatives, $0 \%$ falsenegative, and $0 \%$ false-positive). The cumulative gain analysis demonstrates excellent positive performance against random classification, with a maximum gain score of 0.983 .

\section{DISCUSSION}

Partial extravasation of the patient dose, even with complete resolution during the uptake period, significantly changes the radiopharmaceutical kinetics and impacts the accuracy of the SUV. This effect arises because of the decreased activity imaged 

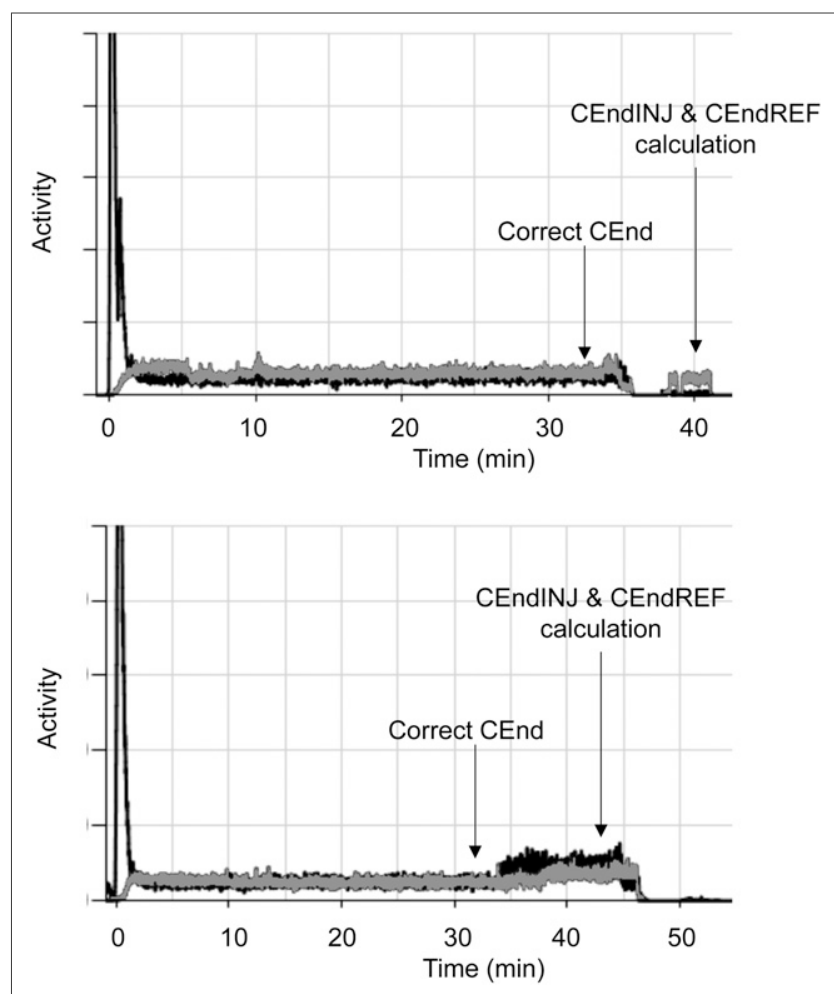

FIGURE 4. Sample ${ }^{68} \mathrm{Ga}-\mathrm{PSMA}$ time-activity curves (with injection curve in black and reference curve in gray) terminated early, with generation of count anomalies erroneously used for CEndINJ and CEndREF calculations. Visual inspection of each timeactivity curve should allow identification and mitigation of risk, including identifying correct CEnd point. Color version of this figure, showing gray curves as red, is available as supplemental file at http://tech.snmjournals.org.

and the trickle effect into the vascular space associated with extravasated injections (3). The precise impact of partial extravasation on SUV will depend on the pharmacokinetics of the infiltration, the proportion of the dose extravasated, and the proportion and rate of any dose that reenters circulation (4). Although the precise impact on image quality and quantitation is difficult to determine (4), a tc50 calculation above $1,200 \mathrm{~s}$ will almost certainly have a deleterious impact on SUV calculation, and-subject to the proportion of the dose extravasated being greater than $5 \%$ - a tc 50 between 600 and 1,200 s may also result in SUV calculation errors but should prompt interpretative caution. A tc50 below $600 \mathrm{~s}$ indicates normality or venous retention, with rapid clearance and negligible impact on SUV. Further investigation and validation of this metric is warranted for this purpose.

For ${ }^{18}$ F-FDG studies, a time-activity curve score above 200 was achieved in only $33.3 \%$ of cases, as likely reflects the lack of validation against parameters used at this site: autoinjector slow bolus with double-flush triple peaks and significantly lower patient doses $(\sim 185 \mathrm{MBq}$ [5 mCi] compared with the $370 \mathrm{MBq}[10 \mathrm{mCi}]$ used for validation of the score metric). For classification of an extravasated dose, a score cutoff of 200 produced a $33 \%$ sensitivity and $96.8 \%$ specificity for the ${ }^{18} \mathrm{~F}-\mathrm{FDG}$ subgroup. No alternative cutoff improved this performance. Clearly, the utility may be improved with metrics that accommodate the broader bolus, slower clearance times, and variations in patient dose. Indeed, the sensitivity and specificity in this study are well short of those previously reported (82\% and $100 \%$, respectively) in studies using ${ }^{18} \mathrm{~F}-\mathrm{FDG}$ and manual injection only (4).

The automatically calculated metrics outlined above are particularly vulnerable to a broader bolus from slower administration, as is reflected in the less-than-optimal predictive performance of metrics dependent on curve behavior in the first $5 \mathrm{~min}$, including the time-activity curve score. Metrics independent of the behavior of the time-activity curve over the first 5 min (e.g., tc50) were demonstrated to be more robust predictors of extravasation-and, indeed, of the ability to differentiate between extravasation and venous retention - and might be readily considered a marker for determining a deleterious impact of injection kinetics on SUV calculation (e.g., the tc50).

\section{aUCR10}

The aUCR10 assumes a tight bolus peaking at $1 \mathrm{~min}$ after injection with rapid clearance, which would produce a nearly 1:1 ratio for a normal injection. Abnormal injections depend on retention of injection sensor activity beyond $1 \mathrm{~min}$ to produce a ratio beyond 2:1. Unfortunately, this metric is skewed by any delay in clearance (e.g., venous retention), by a broader bolus, and by primary or secondary peaks associated with the autoinjector. Although aUCR10 may be less effective for differentiating normal from abnormal vascular retention, it should be sufficiently sensitive to predict extravasation. Because this metric is dose-dependent, variability will occur with different dose activities administered.

\section{aUCR1}

aUCR1 is limited to the period from 60 to $90 \mathrm{~s}$ after injection and, as such, is more susceptible to variations in the time-activity curve associated with the autoinjector and, thus, lower discriminatory power, including for extravasation. Because this metric is dose-dependent, variability will occur with different dose activities administered.

\section{c1}

c1 is the average counts per second recorded by the injection sensor during the interval between 60 and $90 \mathrm{~s}$ after injection. c1 will fluctuate with the dose administered and the radiopharmaceutical. The value of $\mathrm{c} 1$ is reliant on a tight bolus, and thus, the broader bolus of the autoinjector infusion combined with the multiple slow flushes (secondary and tertiary peaks) will undermine the rigor of this metric in predicting variation from normal, as well as the efficacy of differentiating extravasation from venous retention. Because this metric is dose-dependent, variability will occur with different dose activities administered. 


\section{CEndINJ and CEndREF}

CEndINJ and CEndREF are likely susceptible to variations in monitoring time. Indeed, there was a wide variety of monitoring periods ranging from 20 through $60 \mathrm{~min}$. Nonetheless, identifying an endpoint beyond $20 \mathrm{~min}$ should exhibit, in the absence of extravasation, convergence of CEndINJ and CEndREF. Individually, the metrics are not particularly useful as discriminators despite the fact that CEndINJ is higher for extravasation. Because these metrics are dose-dependent, variability will occur with different dose activities administered.

\section{tHalf}

tHalf is a mean half-clearance rate, which should be higher for extravasated injections. Clearance is also delayed for venous retention, and the clearance rate is confounded by the secondary and tertiary peaks associated with the autoinjector. It would be possible to use mathematic curve stripping to extract a single curve representative of clearance, but given that the sensors are not positioned over the injection site itself, it may be more useful to express tHalf as the half-clearance time for a specific period after the normal bolus period. For example, a first tHalf from 5 to $10 \mathrm{~min}$ and a second from 10 to $20 \mathrm{~min}$ would provide more useful metrics to differentiate among normal, venous retention, and extravasated injections.

\section{tc50}

tc50 appears to be the most useful metric for differentiating extravasation from other injection outcomes and perhaps makes the previously suggested 5- to $10-\mathrm{min}$ and $10-$ to 20-min half-clearance calculations redundant. A normal time-activity curve, including in patients with venous retention, should see the injection counts approach the reference counts (to the point of injection counts reducing to within double the reference counts) by $10 \mathrm{~min}$. A tc50 greater than 600 (seconds) suggests abnormality, but a tc50 greater than 1,200 (20 min) may offer a useful marker for certainty of extravasation.

\section{ndAvg1}

ndAvg1 is confounded by the broader peak and secondary and tertiary peaks associated with the autoinjector and in patients exhibiting venous retention. This issue decreases the discriminatory value of this metric.

\section{ndAvgN}

ndAvgN should have good discriminatory power between normal injections (including with autoinjectors) and abnormal injections. Among abnormal injections, there may be difficulty differentiating venous retention from extravasation at 4 min after injection.

\section{Time-Activity Curve Score}

The time-activity curve score is a linear, weighted combination of these metrics. Although the weights are determined from a logistic regression, in the case of the unique characteristics of time-activity curves for the KARL100 autoinjector and Rad-Inject pump, time-activity curve score may be simply multiplying redundancy - that is, multiplying metrics that offer the same insight and using multipliers that lack correlation.
CEnd ratio is a new metric introduced in this project that expresses the relationship of CEndINJ and CEndREF as a ratio. A ratio approximating 1:1 indicates normality at that time (normal injection or rapid resolution of venous retention) and is thought to offer a useful tool for differentiation of extravasation from other anomalies and normal injections.

Several other automated measures that contribute to determination of the time-activity curve score may be better as independent identifiers of extravasation, including tc50 and ndAvgN. Using a tc50 cutoff of $600 \mathrm{~s}, 100 \%$ sensitivity and $100 \%$ specificity for identifying extravasation was noted using both conventional statistical analysis and artificial neural network analysis. That is, if at $20 \mathrm{~min}(1,200 \mathrm{~s})$, the reference curve is less than $50 \%$ of the value of the injection curve, careful consideration should be given to an extravasated injection. As previously discussed, a cutoff of $600 \mathrm{~s}$ provides $100 \%$ sensitivity but only $95 \%$ specificity, with venous retention among the false-positive cases. A cutoff of 25 for ndAvgN demonstrated $100 \%$ sensitivity and $99.7 \%$ specificity for classifying extravasation, although $100 \%$ sensitivity and specificity were noted for ${ }^{18}$ F-FDG studies only. The cutoff of 0 was used to distinguish abnormal time-activity curves (venous retention but excluding extravasation) from normal time-activity curves with a $95.5 \%$ sensitivity and $41.4 \%$ specificity. This project proposed the new metric CEnd ratio, which, using a cutoff of 2 , provides $100 \%$ sensitivity and specificity in classifying extravasation.

The use of topical sensors and automated scoring would add incremental clinical value, with further validation of all metrics against low-dose injections via autoinjectors for a range of radiopharmaceuticals. The predictive power of existing Lucerno Dynamics metrics and those introduced in this project with respective cutoffs also needs to be further validated but could provide deeper insight into injection behavior. The time-activity curves and metrics are sensitive to identification of extravasated injections, thus helping to characterize dose behavior and obviate imaging of the injection site. Specifically, the following are recommended markers but warrant further validation: tc50 greater than 1,200 indicates extravasation and a negative impact on SUV; tc50 less than 600 indicates a normal injection or venous retention that resolves, and no impact on SUV should be expected; tc50 between 600 and 1,200 is not extravasated but needs careful assessment of poor bolus kinetics and the impact on SUV; ndAvgN less than 0 indicates a normal injection; ndAvgN greater than 25 indicates extravasation; ndAvgN between 0 and 25 suggests vascular retention but may be a normal injection; and CEnd ratio greater than 2 indicates extravasation.

Artificial neural network analysis suggested scores generated by multiple metrics compound error and include redundancy, are unnecessarily complex, and increase potential error. Although CEnd ratio and ndAvgN were identified with tc50 as the key variables, with very high correlation, use of these metrics in combination provides no greater predictive capability than tc50 alone-a case of Occam's razor. While 
the neural analysis clarified findings of conventional analysis, the reliability of the neural network is limited by the small positive cohort in the binary analysis.

Identification of the presence of extravasation does not indicate an actual impact on image quality or SUV accuracy. Further research is required to differentiate clinically important extravasation from evidence of clinically insignificant extravasation. The U.S. Nuclear Regulatory Commission has determined, with respect to radiopharmaceutical administration, that extravasation frequently occurs and is virtually impossible to avoid, and the commission does not consider extravasation to be misadministration. As a result, extravasation avoids being subject to the reporting and accountability requirements of misadministration. This study contradicts this position, with use of an autoinjector technique not only reducing staff doses but demonstrating that very low levels of extravasation are possible. Potentially eliminating extravasation for PET studies is possible, and every effort to achieve this reduction is essential to enhance image quality, improve SUV accuracy and reliability, and minimize unintentional exposure of patient tissue to radiation. The recognition of extravasated doses as misadministration, with the associated investigation and reporting requirements of regulators in Australia, may also be a factor in reducing rates.

\section{CONCLUSION}

Topical monitoring and characterization of PET dose administration are possible and practical with the Lara device, with careful consideration and validation of individual metrics. Extravasation and partial extravasation of PET doses are readily detected and differentiated using time-activity curve metrics. The metrics can provide the insight that could inform image quality or SUV accuracy issues. Importantly, extravasation or partial extravasation of PET doses can be minimized with an autoinjector. Further validation of key metrics is recommended in a larger and more diverse (radiopharmaceuticals, injection methods) cohort with discrimination between clinically significant and clinically insignificant extravasation cases.

\section{DISCLOSURE}

This project was funded by an ANSTO/ANZSNM research grant. No other potential conflict of interest relevant to this article was reported.

\section{ACKNOWLEDGMENTS}

This project was undertaken as part of the honors work of Stephanie Sanchez and supervised by Geoffrey Currie. We thank the Lucerno Dynamics team in the United States and the Australian distributors (Bayer) of the Lara device for their patience, encouragement, support, and guidance. We also thank the dedicated staff in the clinical department for supporting and assisting with the project.

\section{REFERENCES}

1. Hillner BE, Siegel BA, Liu D, et al. Impact of positron emission tomography/ computed tomography and positron emission tomography (PET) alone on expected management of patients with cancer. Initial results from the National Oncologic PET Registry. J Clin Oncol. 2008;26:2155-2161.

2. Williams JM, Arlinghaus LR, Rani SD, et al. Towards real-time topical detection and characterization of FDG dose infiltration prior to PET imaging. Eur J Nucl Med Mol Imaging. 2016;43:2374-2380.

3. Lattanze RK, Osman MM, Ryan KA, Frye S, Townsend DW. Usefulness of topically applied sensors to assess the quality of ${ }^{18} \mathrm{~F}-\mathrm{FDG}$ injections and validation against dynamic positron emission tomography (PET) images. Front Med (Lausanne). 2018;5:303.

4. Wong TZ, Benefield T, Masters S, et al. Quality improvement initiatives to assess and improve PET/C injection infiltration rates at multiple centers. J Nucl Med Technol. 2019;47:326-331.

5. Osman MM, Muzaffar R, Altinyay ME, Teymouri C. FDG dose extravasations in PET/CT: frequency and impact on SUV measurements. Front Oncol. 2011;1:41.

6. Silva-Rodríguez J, Aguiar P, Sánchez M, et al. Correction for FDG PET dose extravasations: Monte Carlo validation and quantitative evaluation of patient studies. Med Phys. 2014;41:052502.

7. Muzaffar R, Frye SA, McMunn A, Ryan K, Lattanze R, Osman MM. Novel method to detect and characterize ${ }^{18} \mathrm{~F}-\mathrm{FDG}$ infiltration at the injection site: a single-institution experience. J Nucl Med Technol. 2017;45:267-271.

8. Currie GM. Intelligent imaging: artificial intelligence augmented nuclear medicine. J Nucl Med Technol. 2019;47:217-222.

9. Currie G. Intelligent imaging: anatomy of machine learning and deep learning. $J$ Nucl Med Technol. 2019;47:273-281.

10. Currie G, Hawk KE, Rohren E, Vial A, Klein R. Machine learning and deep learning in medical imaging: intelligent imaging. J Med Imaging Radiat Sci. 2019;50:477-487. 